\title{
Some remarks on limit mixed Hodge structures and spectrum
}

\author{
Alexandru Dimca and Morihiko Saito
}

\begin{abstract}
In this note we clarify some subtle points on the limit mixed Hodge structures and on the spectrum. These are more or less well-known to the specialists, but do not seem to be stated explicitly in the literature. However, as they do not seem to be obvious to the beginners, we consider them to be worth writing down explicitly. The general constructions are exemplified by considering the isolated (weighted) homogeneous singularities in detail.
\end{abstract}

\section{Limit mixed Hodge structure}

1.1. In [19], [23], the limit mixed Hodge structures were constructed in the unipotent monodromy case. For the non-unipotent case, we can combine it with [20] as follows. Here we describe the limit of the mixed Hodge structure on the cohomology with compact supports using the Cech-type construction, since this seems to be the easiest way to explain the relation with the theory of motivic Milnor fibers [4].

For the usual cohomology (i.e. without compact supports), we can use the commutativity of the dualizing functor $\mathbf{D}$ and the passage to the limit mixed Hodge structure, i.e. $\mathbf{D} \circ \psi_{t}=\psi_{t} \circ \mathbf{D}$ (up to a Tate twist), see [23], [15] as well as [6] for generalities on constructible or perverse sheaves. Of course, we can also use the two weight filtrations on the logarithmic complex associated with the divisor with $V$-normal crossings [20] as in [23]. It also follows from the theory of mixed Hodge modules [14], [15].

Key Words: limit mixed Hodge structure, monodromy, Milnor fiber, spectrum.

2010 Mathematics Subject Classification: Primary 14D07, 32S35; Secondary 14F05,32S40. Received: 16 October 2012.

Accepted: 5 November 2012. 
Let $f: X \rightarrow \Delta$ be a projective morphism of complex manifolds where $\Delta$ is an open disk. We may assume that $f$ is smooth over $\Delta^{*}$ (shrinking $\Delta$ if necessary). Set $Y:=f^{-1}(0)$. Let $D$ be a divisor on $X$ which is flat over $\Delta$, i.e. all the irreducible components $D_{j}$ of $D$ are dominant over $\Delta$. Assume $D \cup Y$ is a divisor with simple normal crossings. Set

$$
U:=X \backslash D, \quad f^{\prime}:=\left.f\right|_{U}: U \rightarrow \Delta, \quad D_{J}:=\bigcap_{j \in J} D_{j} \quad\left(\text { where } D_{\emptyset}=X\right) .
$$

Let $Y_{i}$ be the irreducible components of $Y \subset X$ with $m_{i}$ the multiplicity of $Y$ along the generic point of $Y_{i}$. Set $m=\operatorname{LCM}\left(m_{i}\right)$. Let $\tilde{f}: \widetilde{X} \rightarrow \widetilde{\Delta}$ be the normalization of the base change of $f: X \rightarrow \Delta$ by the ramified $m$-fold covering $\pi_{\Delta}: \widetilde{\Delta} \rightarrow \Delta$ which is finite étale over $\Delta^{*}$, where $\widetilde{\Delta}$ is an open disk. Let $\pi: \widetilde{X} \rightarrow X$ be the canonical morphism. Set

$$
\widetilde{U}:=\pi^{-1}(U), \quad \widetilde{Y}:=\pi^{-1}(Y), \quad \widetilde{D}:=\pi^{-1}(D), \quad \widetilde{D}_{J}:=\pi^{-1}\left(D_{J}\right) .
$$

Then $\widetilde{X}$ is a $V$-manifold, and $\widetilde{Y} \cup \widetilde{D}$ is a divisor with $V$-normal crossings on $\widetilde{X}$. Let $\tilde{j}: \widetilde{U} \hookrightarrow \widetilde{X}$ be the natural inclusion. There is a natural quasi-isomorphism

$$
\tilde{j}_{!} \mathbf{Q}_{\widetilde{U}} \stackrel{\sim}{\longrightarrow} \mathcal{K}_{\tilde{X}}^{\bullet} \quad \text { with } \quad \mathcal{K}_{\widetilde{X}}^{p}:=\bigoplus_{|J|=p} \mathbf{Q}_{\widetilde{D}_{J}},
$$

where the differential of $\mathcal{K}_{\tilde{X}}^{\bullet}$ is defined in the same way as a Cech complex as is well known.

Consider the complex

$$
\psi_{\tilde{f}} \mathcal{K}_{\widetilde{X}}^{\bullet}
$$

This naturally underlies a cohomological mixed Hodge complex such that its restriction to $\psi_{\tilde{f}} \mathbf{Q}_{\widetilde{D}_{J}}$ coincides with the one defined in [St2] using the complex of logarithmic forms $\widetilde{\Omega}_{\widetilde{D}_{J}}\left(\log \left(\widetilde{Y} \cap \widetilde{D}_{J}\right)\right)$ together with the Hodge filtration $F$ and the weight filtration $W$ on it. Indeed, we have canonical morphisms for $J \subset J^{\prime}$

$$
\left.\widetilde{\Omega}_{\widetilde{D}_{J}}^{\bullet}\left(\log \left(\widetilde{Y} \cap \widetilde{D}_{J}\right)\right)\right|_{\widetilde{D}_{J^{\prime}}} \rightarrow \widetilde{\Omega}_{\widetilde{D}_{J^{\prime}}}\left(\log \left(\widetilde{Y} \cap \widetilde{D}_{J^{\prime}}\right)\right),
$$

and this is a filtered quasi-isomorphism for $W$ (forgetting the filtration $F$ ), since $D \cup Y$ is a divisor with normal crossings.

There is a spectral sequence of mixed Hodge structures

$$
{ }_{\infty} E_{1}^{p, q}=\bigoplus_{|J|=p} H^{q}\left(\widetilde{D}_{J, \infty}, \mathbf{Q}\right) \Longrightarrow H_{c}^{p+q}\left(\widetilde{U}_{\infty}, \mathbf{Q}\right),
$$

which is induced by the truncations $\tau_{\geq k}$ on $\mathcal{K}_{\widetilde{X}}^{\bullet}$ for $k \in \mathbf{Z}$, and degenerates at $E_{2}$. (This is the dual of the spectral sequence in [StZ], 5.7.) Indeed, it is the 'limit' by $\rightarrow 0$ of the weight spectral sequence

$$
E_{1}^{p, q}=\bigoplus_{|J|=p} H^{q}\left(\widetilde{D}_{J}, \mathbf{Q}\right) \Longrightarrow H_{c}^{p+q}(\widetilde{U}, \mathbf{Q}),
$$


where $\widetilde{X}:=\tilde{f}^{-1}()$ and $\widetilde{D}_{J}:=\widetilde{D}_{J} \cap \widetilde{X}$ for $\in \widetilde{\Delta}^{*}$. These spectral sequences are the dual of the spectral sequences in [23] in the unipotent monodromy case.

Note that (1.1.2) is compatible with the actions of the semisimple part $T_{s}$ and the nilpotent part $N:=(2 \pi i)^{-1} \log T_{u}$ of the monodromy $T$.

1.2. The relation with motivic nearby fibers. With the above notation, let $E=Y \cup D$ with $E_{i}$ the irreducible components of $E$. We may assume $E_{i}=Y_{i}$ for $i \leq r$ and $E_{i}=D_{i-r}$ for $i>r$, where $r$ is the number of the irreducible components of $Y$. For $I$ with $\min (I) \leq r$ (i.e. $E_{I} \subset Y$ ), define $(1.2 .1)$

$$
E_{I}=\bigcap_{i \in I} E_{i}, \quad E_{I}^{\circ}=\bigcap_{i \in I} E_{i} \backslash \bigcap_{i \notin I} E_{i}, \quad \widetilde{E}_{I}=\pi^{-1}\left(E_{I}\right), \quad \widetilde{E}_{I}^{\circ}=\pi^{-1}\left(E_{I}^{\circ}\right) .
$$

Note that $\widetilde{E}_{I}^{\circ} \rightarrow E_{I}^{\circ}$ is a cyclic étale covering. Set $I^{\prime}:=I \cap[1, r]$. Let $\mathbf{L}$ denote $\mathbf{1}(-1)$ as an Chow motive where $\mathbf{1}=[p t]$, and $(-1)$ is the Tate twist, see e.g. $[\mathrm{Mu}],[\mathrm{Sch}]$. By $[\mathrm{DL}],[\mathrm{Lo}]$ and $[\mathrm{MT}],[\mathrm{Ra}]$, the motivic nearby fibers for the morphisms $f: X \rightarrow \Delta$ and $f^{\prime}: U \rightarrow \Delta$ can be given respectively by

$$
\text { (1.2.2) } \sum_{\min (I) \leq r}\left[\left(\widetilde{E}_{I}^{\circ}, T_{s}\right)\right](\mathbf{1}-\mathbf{L})^{\left|I^{\prime}\right|-1}, \quad \sum_{\max (I) \leq r}\left[\left(\widetilde{E}_{I}^{\circ}, T_{s}\right)\right](\mathbf{1}-\mathbf{L})^{|I|-1} .
$$

These belong to the Grothendieck group of Chow motives (with Q-coefficients) endowed with an action of $T_{s}$ of finite order by using equivariant resolutions of $\left(\widetilde{E}_{I}, \widetilde{E}_{I} \backslash \widetilde{E}_{I}^{\circ}\right)$, see [DL]. Here $T_{s}$ denotes the semi-simple part of the monodromy, and is given by the automorphism $\gamma$ of $\widetilde{X}$ over $X$ induced by the base change of the automorphism of $\widetilde{\Delta}$ defined by $\mapsto \zeta_{\mathrm{m}}$ with $\zeta_{m}:=\exp (2 \pi i / m)$. The action of $T_{s}$ on $\mathbf{L}$ is the identity. We denote the images of the two terms of (1.2.2) in the Grothendieck group of mixed Hodge structures with an action of finite order respectively by

$\sum_{\min (I) \leq r}\left[\left(H_{c}^{\bullet}\left(\widetilde{E}_{I}^{\circ}\right), T_{s}\right](\mathbf{1}-\mathbf{L})^{\left|I^{\prime}\right|-1}, \quad \sum_{\max (I) \leq r}\left[\left(H_{c}^{\bullet}\left(\widetilde{E}_{I}^{\circ}\right), T_{s}\right)\right](\mathbf{1}-\mathbf{L})^{|I|-1}\right.$,

where $H_{c}^{\bullet}\left(\widetilde{E}_{I}^{\circ}\right)$ is a complex of mixed Hodge structures with zero differential, $\mathbf{L}$ means here the class of $\mathbf{Q}(-1)$ with trivial action of the monodromy, and $T_{s}$ is given by $\left(\gamma^{*}\right)^{-1}$, see (2.1.2) below. Then these respectively coincide in the notation of (1.1.1) with

$$
\left[\left(H^{\bullet}\left(\widetilde{X}_{\infty}\right), T_{s}\right)\right], \quad\left[\left(H_{c}^{\bullet}\left(\widetilde{U}_{\infty}\right), T_{s}\right)\right] .
$$

Indeed, this follows from the construction of Steenbrink [20] together with the long exact sequence of mixed Hodge structures

$$
\rightarrow H_{c}^{j}\left(Z^{\prime}\right) \rightarrow H_{c}^{j}(Z) \rightarrow H_{c}^{j}\left(Z \backslash Z^{\prime}\right) \rightarrow H_{c}^{j+1}\left(Z^{\prime}\right) \rightarrow,
$$

for any open immersions of complex algebraic varieties $Z^{\prime} \hookrightarrow Z$, which is compatible with the action of automorphisms of varieties. (Here (1.2.5) can 
be proved by using mixed Hodge modules or the mapping cone construction in [3] together with the diagram of the octahedral axiom of derived categories.) The dual exact sequence of (1.2.5) for Borel-Moore homology is well known in the theory of cycle maps of higher algebraic cycles.

Note that we get cohomology with compact supports in (1.2.4), and this is quite different from the case of motivic Milnor fibers in [4].

1.3. Remarks. (i) In case $E_{I}$ is simply connected, the cyclic étale covering $\widetilde{E}_{I}^{\circ} \rightarrow E_{I}^{\circ}$ can be determined by the multiplicities $m_{j}$ of $Y$ along the irreducible components $Y_{j}$ intersecting $E_{I}$. For example, assume $\max (I) \leq r$ and

$$
E_{I}=\bigcap_{i \in I} Y_{i}=\mathbf{P}^{1}, \quad E_{I}^{\circ}=E_{I} \backslash\left(E_{i^{\prime}} \cup E_{i^{\prime \prime}}\right)=\mathbf{C}^{*},
$$

with $i^{\prime} \leq r$ (i.e. $E_{i^{\prime}}=Y_{i^{\prime}}$ ). Then the covering degree of $\widetilde{E}_{I}^{\circ} \rightarrow E_{I}^{\circ}$ and the number of connected components of $\widetilde{E}_{I}^{\circ}$ are given respectively by

$$
\operatorname{GCD}\left(m_{i} \mid i \in I\right), \quad \operatorname{GCD}\left(m_{i} \mid j \in I \cup\left\{i^{\prime}\right\}\right) .
$$

This may simplify some argument in [8].

(ii) In [4], the semisimple part of the monodromy $T_{s}$ acts as an automorphism of Chow motives. This seems to be useful for the proof of the independence of the motivic Milnor fiber by the resolutions of singularities. For instance, we have $\left[\mathbf{P}^{1}\right]=\mathbf{1} \oplus \mathbf{1}(-1)$ with $\operatorname{End}(\mathbf{1})=\operatorname{End}(\mathbf{1}(-1))=\mathbf{Q}$ in the category of Chow motives. This is a special case of the Chow-Künneth decomposition, see e.g. [9], [18].

\section{Spectrum}

2.1. Geometric monodromy and local system monodromy. Let $f$ : $X \rightarrow S$ be a continuous map of topological spaces which is locally topologically trivial over $S$. We assume that the $H_{j}\left(X_{s}\right)$ and $H^{j}\left(X_{s}\right)$ with Q-coefficients are finite dimensional for any $j$. Let $s \in S$, and $\gamma \in \pi_{1}(S, s)$. Let $\rho: Y \rightarrow[0,1]$ be the base change of $f$ by the loop $\gamma$. Choosing a trivialization over $[0,1]$, we get the geometric monodromy

$$
\gamma_{\#}: X_{s}=Y_{0} \simeq Y_{1}=X_{s},
$$

where the middle homeomorphism is induced by the trivialization. We have the induced action of the geometric monodromy on homology and cohomology (with $\mathbf{Q}$-coefficients):

$$
\gamma_{*} \in \operatorname{Aut}\left(H_{j}\left(X_{s}\right)\right), \quad \gamma^{*} \in \operatorname{Aut}\left(H^{j}\left(X_{s}\right)\right),
$$


such that

$$
\gamma^{*}={ }^{t} \gamma_{*}
$$

where ${ }^{t}$ means the transpose.

On the other hand, we have the local system monodromies

$$
\gamma_{h} \in \operatorname{Aut}\left(H_{j}\left(X_{s}\right)\right), \quad \gamma_{c} \in \operatorname{Aut}\left(H^{j}\left(X_{s}\right)\right),
$$

which are defined by using the following (trivial) local systems of homology and cohomology groups over $[0,1]$ :

$$
\left\{H_{j}\left(Y_{u}\right)\right\}_{u \in[0,1]}, \quad\left\{H^{j}\left(Y_{u}\right)\right\}_{u \in[0,1]} .
$$

The latter can be identified with the constant sheaf $R^{j} \rho_{*} \mathbf{Q}_{Y}$, see also [2], XIV, 1.1.2. Note that $\gamma_{c}$ coincides with the monodromy associated to the nearby cycle functor if $f$ is a Milnor fibration.

The relations between the above monodromies are given by

$$
\gamma_{*}=\gamma_{h}, \quad \gamma^{*}=\gamma_{c}^{-1} .
$$

Indeed, the first assertion easily follows from the definition (using simplicial chains for example). We then get the second equality since

$$
\gamma^{*}={ }^{t} \gamma_{*}={ }^{t} \gamma_{h}=\gamma_{c}^{-1},
$$

where the last equality follows from

$$
\left\langle\gamma_{c} u, \gamma_{h} v\right\rangle=\langle u, v\rangle \quad \text { for } u \in H^{j}\left(X_{s}\right), v \in H_{j}\left(X_{s}\right) .
$$

Here $\langle u, v\rangle$ denotes the canonical pairing between cohomology and homology, and it can be extended to a canonical pairing between the local systems so that (2.1.3) follows.

It does not seem that (2.1.2) has been clarified explicitly in the literature. In fact, it does not seem to cause big problems at least in the local monodromy case since it is quasi-unipotent (except possibly for the definition of spectrum as in [8]).

2.2. Example. Let $f$ be a homogeneous polynomial of $n$ variables with degree $d$, having an isolated singularity at the origin. Set $X=\mathbf{C}^{n} \backslash f^{-1}(0)$ and $S=\mathbf{C}^{*}$. Here $f$ also denotes the morphism $X \rightarrow S$ induced by $f$. Let $\gamma$ be a generator of $\pi_{1}(S, s) \simeq \mathbf{Z}$ going around the origin counter-clockwise. Then $\gamma_{\#}$ is induced by the automorphism

$$
\gamma_{\#}:\left(x_{1}, \ldots, x_{n}\right) \mapsto\left(\zeta_{d} x_{1}, \ldots, \zeta_{d} x_{n}\right),
$$


where $\zeta_{d}:=\exp (2 \pi i / d)$, and $x_{1}, \ldots, x_{n}$ are the coordinates of $\mathbf{C}^{n}$. The action of $\gamma_{\#}$ is extended to an automorphism of $\mathbf{C}\left[x_{1}, \ldots, x_{n}\right]$ over $\mathbf{C}$ such that

$$
\gamma_{\#}^{*} x_{i}=\zeta_{d} x_{i} .
$$

This can be checked for instance by $\gamma_{\#}^{*}\left(x_{i}-\zeta_{d} c_{i}\right)=\zeta_{d}\left(x_{i}-c_{i}\right)$.

Set $\omega=d x_{1} \wedge \cdots \wedge d x_{n}$, and

$$
H_{f}^{\prime \prime}:=\Omega_{X, 0}^{n} / d f \wedge d \Omega_{X, 0}^{n-2} .
$$

This is called the Brieskorn lattice. Let $g \in \mathbf{C}\left[x_{1}, \ldots, x_{n}\right]$ be a monomial of degree $k$. After Brieskorn, it is well known (and easy to show) that

$$
\partial_{t} t(g \omega)=\frac{k+n}{d} g \omega \text { in } H_{f}^{\prime \prime},
$$

see e.g. the proof of Prop. 3.3 in [13] for an argument in a slightly more general case.

In the homogeneous polynomial case, we have moreover the well-known relation

$$
\gamma_{c}=\exp \left(-2 \pi i\left(\operatorname{Res} t \partial_{t}\right)\right)
$$

under the canonical isomorphism

$$
H^{n-1}\left(X_{1}, \mathbf{C}\right)=H_{f}^{\prime \prime} / t H_{f}^{\prime \prime},
$$

where $s=1$. The isomorphism can be defined by using a basis $\left(\omega_{1}, \ldots, \omega_{\mu}\right)$ of $H_{f}^{\prime \prime}$ such that $\partial_{t} t \omega_{i}=\alpha_{i} \omega_{i}$, and taking the restriction to $X_{1}$ after dividing the $\omega_{i}$ by $d f$. Indeed, the assertion is well known if the Brieskorn lattice is replaced by the Deligne extension [1]. In this case, the inverse isomorphism is given by

$$
u \mapsto \exp \left(-\frac{\log t}{2 \pi i} \log \gamma_{c}\right) u,
$$

for $u \in H^{n-1}\left(X_{1}\right)$ which is identified with a multivalued section, and we have

$$
t \partial_{t} \exp \left(-\frac{\log t}{2 \pi i} \log \gamma_{c}\right) u=-\frac{\log \gamma_{c}}{2 \pi i} \exp \left(-\frac{\log t}{2 \pi i} \log \gamma_{c}\right) u,
$$

where the eigenvalues of $-\frac{1}{2 \pi i} \log \gamma_{c}$ are chosen corresponding to the Deligne extension. This can be extended to the Brieskorn lattice case easily in the homogeneous polynomial case. So $(2.2 .5)$ follows.

By (2.2.4) and (2.2.5), the action of $\gamma_{c}$ on $\left.(g / d f)\right|_{X_{1}} \in H^{n-1}\left(X_{1}, \mathbf{C}\right)$ is given by the multiplication by

$$
\exp (-2 \pi i(k+n) / d)
$$


On the other hand, (2.2.2) implies that the action of the geometric monodromy $(2.2 .1)$ on $\left.(g / d f)\right|_{X_{1}}$ is given by the multiplication by

$$
\exp (2 \pi i(k+n) / d) \text {. }
$$

This is the inverse of (2.2.7).

2.3. Brieskorn lattices and mixed Hodge structures. The Brieskorn lattice $H_{f}^{\prime \prime}$ in (2.2.3) is defined for any holomorphic function on a complex manifold $X$ having an isolated singularity at $0 \in f^{-1}(0)$. It is a free $\mathbf{C}\left\{\left\{\partial_{t}^{-1}\right\}\right\}$ module of rank $\mu$, and is contained in the Gauss-Manin system $\mathcal{G}_{f}$ which is the localization of $H_{f}^{\prime \prime}$ by $\partial_{t}^{-1}$, i.e. $\mathcal{G}_{f}=H_{f}^{\prime \prime}\left[\partial_{t}\right]$. The latter has the Hodge filtration defined by $F^{p} \mathcal{G}_{f}:=\partial_{t}^{n-1-p} H_{f}^{\prime \prime}$ for $p \in \mathbf{Z}$, and also the filtration $V$ of Kashiwara and Malgrange such that $\partial_{t} t-\alpha$ is nilpotent on $\operatorname{Gr}_{V}^{\alpha} \mathcal{G}_{f}$. By an argument similar to the proof of (2.2.6), there are isomorphisms

$$
H^{n-1}\left(X_{f, 0}, \mathbf{C}\right)_{\lambda}=\operatorname{Gr}_{V}^{\alpha} \mathcal{G}_{f} \quad \text { for } \lambda=\exp (-2 \pi i \alpha)
$$

where $X_{f, 0}$ is the Milnor fiber, and $V_{\lambda}$ denotes the $\lambda$-eigenspace for any vector space $V$ with the action of the local system monodromy $T$. We have moreover (2.3.2)

$$
F^{n-1-q} H^{n-1}\left(X_{f, 0}, \mathbf{C}\right)_{\lambda}=\operatorname{Gr}_{V}^{\alpha} H_{f}^{\prime \prime} \text { for } q<\alpha \leq q+1, \quad \lambda=\exp (-2 \pi i \alpha),
$$

where $F$ is the Hodge filtration of the mixed Hodge structure [20], see [17], [24], etc. This is closely related with the definition of the spectrum in $(2.4 .5)$ below. In the case of Example (2.2), it is related with [21] by (2.2.4).

2.4. Spectrum. Let $H$ be a mixed Hodge structure with a semisimple action $T$ of finite order. Set $H_{\mathbf{C}, \lambda}:=\operatorname{Ker}(T-\lambda) \subset H_{\mathbf{C}}$. We define the spectrum $\mathrm{Sp}^{\prime}(H, T)$ as in [Sai4] (and [DL]) by

$$
\begin{aligned}
& \operatorname{Sp}^{\prime}(H, T):=\sum_{\alpha \in \mathbf{Q}} n_{\alpha}^{\prime} t^{\alpha}, \\
& \text { with } \quad n_{\alpha}^{\prime}=\operatorname{dim}_{\mathbf{C}} \operatorname{Gr}_{F}^{p} H_{\mathbf{C}, \lambda} \text { for } p=[\alpha], \lambda=\exp (2 \pi i \alpha) .
\end{aligned}
$$

For a holomorphic function $f$ on a complex manifold $X$ of dimension $n$ and $x \in f^{-1}(0)$, we first define $\operatorname{Sp}^{\prime}(f, x)$ by

$$
\operatorname{Sp}^{\prime}(f, x):=\sum_{j}(-1)^{n-1-j} \operatorname{Sp}^{\prime}\left(\widetilde{H}^{j}\left(X_{f, x}\right), T_{s}\right),
$$

where $\widetilde{H}^{j}\left(X_{f, x}\right)$ is the reduced Milnor cohomology endowed with the canonical mixed Hodge structure, and $T_{s}$ is the semisimple part of the local system monodromy $T$. There are canonical isomorphisms

$$
\widetilde{H}^{j}\left(X_{f, x}\right)=H^{j} i_{x}^{*} \varphi_{f} \mathbf{Q}_{X},
$$


where $i_{x}:\{x\} \hookrightarrow X$ is the inclusion, see [2]. They can be used to define the mixed Hodge structure on the left-hand side. Note that $T$ is equal to the inverse of the cohomological Milnor monodromy by (2.1), and this is closely related with Example (2.2) by (2.3).

Let $\iota$ denote the involution of $\mathbf{Z}\left[t^{1 / m}, t^{-1 / m}\right]$ over $\mathbf{Z}$ defined by

$$
\iota\left(t^{\alpha}\right)=t^{-\alpha} \text {. }
$$

The spectrum $\operatorname{Sp}(f, x)$ is then defined by

$$
\operatorname{Sp}(f, x):=t^{n} \iota\left(\operatorname{Sp}^{\prime}(f, x)\right) .
$$

This spectrum $\operatorname{Sp}(f, x)$ coincides with the one in [20] for the isolated singularity case (using the complex conjugate of (2.4.1) together with the symmetry (2.4.6) below). It coincides with the one in [22] up to the multiplication by $t$. Indeed, the above definition of $\operatorname{Sp}(f, x)$ can be rewritten as $\operatorname{Sp}(f, x):=\sum_{\alpha \in \mathbf{Q}} n_{\alpha} t^{\alpha}$ with

$$
\begin{aligned}
& n_{\alpha}=\sum_{j}(-1)^{n-1-j} \operatorname{dim}_{\mathbf{C}} \operatorname{Gr}_{F}^{n-1-q} \widetilde{H}^{j}\left(X_{f, x}, \mathbf{C}\right)_{\lambda} \\
& \text { for } q<\alpha \leq q+1, \lambda=\exp (-2 \pi i \alpha),
\end{aligned}
$$

and this is used in loc. cit. (up to the multiplication by $t$ ). In the isolated singularity case, the formula (2.4.5) is closely related with (2.3.2) and also with the calculations in (2.2).

If $f$ has an isolated singularity at $x$, we have the symmetry of mixed Hodge numbers by [20] so that

$$
\operatorname{Sp}(f, x)=\operatorname{Sp}^{\prime}(f, x) .
$$

In case $f$ is a weighted-homogeneous polynomial of weights $\left(w_{1}, \ldots, w_{n}\right)$ (i.e. $f$ is a linear combination of monomials $x_{1}^{m_{1}} \cdots x_{n}^{m_{n}}$ with $\sum_{i} w_{i} m_{i}=1$ ) and has an isolated singularity at 0 , it is well known that

$$
\operatorname{Sp}(f, 0)=\prod_{i=1}^{n}\left(\frac{t-t^{w_{i}}}{t^{w_{i}}-1}\right) .
$$

This follows from [21], see also [20] and the proof of Proposition 5.2 in [5]. In the case of homogeneous polynomials (i.e. $w_{i}=1 / d$ for any $i$ ), this follows also from the calculation in Example (2.2) using (2.3).

For a polynomial mapping $f: \mathbf{C}^{n} \rightarrow \mathbf{C}$, we can define the spectrum at infinity (see also [11]) by

$$
\begin{aligned}
\operatorname{Sp}^{\prime}(f, \infty) & :=\sum_{j}(-1)^{n-1-j} \operatorname{Sp}^{\prime}\left(\widetilde{H}^{j}\left(X_{\infty}\right), T_{s}\right), \\
\operatorname{Sp}(f, \infty) & :=t^{n} \iota\left(\operatorname{Sp}^{\prime}(f, \infty)\right),
\end{aligned}
$$


where $\widetilde{H}^{j}\left(X_{\infty}\right)$ is the limit mixed Hodge structure of $\widetilde{H}^{j}\left(X_{t}\right)$ at infinity (of $\mathbf{C}$ ), and $T_{s}$ is the semisimple part of the local system monodromy $T$ associated with a sufficiently large loop around the origin which goes counter-clockwise from the origin (and clockwise from $\infty \in \mathbf{P}^{1}$ ). This definition is compatible with the one in the weighted-homogeneous isolated singularity case in (2.4.7). In the cohomologically tame case, we have the symmetry by [12] (i.e. Theorem 1 in this paper) so that (2.4.6) holds, and the definition (2.4.8) seems to coincide with the one in [8] (where the cohomology with compact supports is used) if the local system monodromy is used there.

\section{References}

[1] Deligne, P., Equation différentielle à points singuliers réguliers, Lect. Notes in Math. 163, Springer, Berlin, 1970.

[2] Deligne, P., Le formalisme des cycles évanescents, in SGA7 XIII and XIV, Lect. Notes in Math. 340, Springer, Berlin, 1973, pp. 82-115 and 116-164.

[3] Deligne, P., Théorie de Hodge, III, Publ. Math. I.H.E.S. 44 (1974), 5-77.

[4] Denef, J. and Loeser, F., Motivic Igusa zeta functions, J. Alg. Geom. 7 (1998), 505-537.

[5] Dimca, A., Monodromy and Hodge theory of regular functions, in New Developments in Singularity Theory, Kluwer Acad. Publ., Dordrecht, 2001, pp. 257-278.

[6] Dimca, A., Sheaves in Topology, Universitext, Springer, 2004.

[7] Looijenga, E., Motivic measures, in Séminaire Bourbaki 1999/2000, Astérisque 276 (2002), 267-297.

[8] Matsui, Y. and Takeuchi, K., Monodromy at infinity of polynomial maps and Newton polyhedra, preprint (arXiv:0912.5144v11).

[9] Murre, J.P., On the motive of an algebraic surface, J. reine angew. Math. 409 (1990), 190-204.

[10] Raibaut, M., Fibre de Milnor motivique à l'infini, C. R. Acad. Sci. Paris, Ser. I 348 (2010), 419-422.

[11] Sabbah, C., Monodromy at infinity and Fourier transform, Publ. RIMS, Kyoto Univ. 33 (1997), 643-685. 
[12] Sabbah, C., Hypergeometric periods for a tame polynomial, Port. Math., 63 (2006), 173-226 (or arXiv:math/9805077).

[13] Saito, M., Exponents and Newton polyhedra of isolated hypersurface singularities, Math. Ann. 281 (1988), 411-417.

[14] Saito, M., Modules de Hodge polarisables, Publ. RIMS, Kyoto Univ. 24 (1988), 849-995.

[15] Saito, M., Mixed Hodge modules, Publ. RIMS, Kyoto Univ. 26 (1990), $221-333$.

[16] Saito, M., On Steenbrink's conjecture, Math. Ann. 289 (1991), 703-716.

[17] Scherk, J. and Steenbrink, J.H.M., On the mixed Hodge structure on the cohomology of the Milnor fibre, Math. Ann. 271 (1985), 641-665.

[18] Scholl, A.J. , Classical Motives, Proc. Symp. Pure Math. 55 (1994), Part $1,163-187$.

[19] Steenbrink, J.H.M., Limits of Hodge structures, Inv. Math. 31 (1976), 229-257.

[20] Steenbrink, J.H.M., Mixed Hodge structure on the vanishing cohomology, in Real and complex singularities, Sijthoff and Noordhoff, Alphen aan den Rijn, 1977, pp. 525-563.

[21] Steenbrink, J.H.M., Intersection form for quasi-homogeneous singularities, Compositio Math. 34 (1977), 211-223.

[22] Steenbrink, J.H.M., The spectrum of hypersurface singularities, Astérisque 179-180 (1989), 163-184.

[23] Steenbrink, J.H.M. and Zucker, S., Variation of mixed Hodge structure I, Inv. Math., 80 (1985), 489-542.

[24] Varchenko, A.N., Asymptotic Hodge structure in the vanishing cohomology, Math. USSR Izv. 18 (1982), 469-512.

Alexandru DIMCA

Institut Universitaire de France et

Laboratoire J.A. Dieudonné, UMR du CNRS 7351,

Université de Nice-Sophia Antipolis, Parc Valrose,

06108 Nice Cedex 02, France

Email: dimca@unice.fr

Morihiko SAITO

RIMS Kyoto University, Kyoto 606-8502, Japan

Email: msaito@kurims.kyoto-u.ac.jp 